\title{
Sclerema Neonatorum
}

National Cancer Institute

\section{Source}

National Cancer Institute. Sclerema Neonatorum. NCI Thesaurus. Code C35009.

A diffuse hardening of skin and subcutaneous adipose tissue, associated with minimal inflammation without fat necrosis, that typically affects critically ill preterm neonates during the first week of life. 